\title{
Empiric k·p Hamiltonian calculation of the band-to-band photon absorption in semiconductors
}

\author{
Antonio Luque ${ }^{1,2}$, Aleksandr Panchak ${ }^{1}$, Alexander Mellor ${ }^{1}$, Alexey Vlasov ${ }^{1}$, Antonio \\ Martí ${ }^{2}$ and Viatcheslav Andreev ${ }^{1}$
}

1: Ioffe Physical Technical Institute, 26, Politekhnicheskaya str. St. Petersburg 194021, Russia 2: Solar Energy Institute, Technical University of Madrid, 28040 Madrid, Spain

Corresponding author e-mail: a.luque@upm.es

The Empiric k.p Hamiltonian method is usually applied to nanostructured semiconductors. In this paper, it is applied to a homogeneous semiconductor in order to check the adequacy of the method. In this case, the solutions of the diagonalized Hamiltonian, as well as the envelope functions, are plane waves. The procedure is applied to the GaAs and the interband absorption coefficients are calculated. They result in reasonable agreement with the measured values, further supporting the adequacy of the Empiric k.p Hamiltonian method.

\section{Introduction}

The k.p method introduced by Dresselhaus Kip and Kittel [1] was extensively developed by Kane [2,3] for calculations of semiconductor band structures. The topic is taught in several books [4,5] including its application to nanostructured semiconductors. Even today, with the extensive use of modern computing facilities [6] the $\mathrm{k} \cdot \mathrm{p}$ methods are widely used and new books on the topic are still published [7-9]. However, they contain a background that is not often at the reach of device engineers. A book by Datta [10] (see chapter 6) bridges this gap.

The Empiric k.p Hamiltonian (EKPH) has been proposed [11,12] to perform easy and fast quantum calculations associated to the light absorption of nanostructured semiconductor devices [13-15], primarily, solar cells. By providing a simple means of calculating energy levels and photon-induced-transition probabilities, the method has permitted realistic detailed balance modeling of the subbandgap quantum efficiency of quantum dot (QD) solar cells [16], yielding an unprecedented semiquantitative interpretation of its temperature dependence.

In this paper, we further validate the model by presenting a calculation of the band-to-band absorption coefficient in GaAs based on the EKPH, and comparing to the well-known experimental results in the literature. The purpose is not to contribute to the understanding of the mechanisms involved: band-to-band absorption is one of the fundamental characteristics of semiconductors and is known since the first third of the $20^{\text {th }}$ century (In GaAs it has been measured with precision at least since 1961[17]). Rather, we attempt to give an additional validation of the EKPH calculations with experiments, beyond what has already been shown for interband absorption[11,12,14,16] in order to further justify its use and usefulness for analysis of nanostructured semiconductors.

\section{The EKPH Method}

In the $\mathrm{k} \cdot \mathrm{p}$ methods, the one-electron Hamiltonian is developed in an orthonormal basis formed by the product of the Bloch functions calculated at the semiconductor $\Gamma$ point $(\boldsymbol{k}=0)$ for a certain band and a plane wave of arbitrary wavevector $\boldsymbol{k}$ [10].

In these methods, the stationary wavefunctions $\quad \Xi$ (the eigenfunctions) are expressed as

$$
\Xi(\boldsymbol{r}) \cong \sum_{v} u_{v, 0}(\boldsymbol{r}) \Psi_{v}(\boldsymbol{r})
$$

The so-called envolvents, $\Psi_{v}$, are slowly varying functions that may be considered constant within a given unit cell of the semiconductor crystal. The so-called involutes, $u_{v, 0}$, are the Bloch functions at the $\Gamma$ point; these are periodic with the translational periodicity of the crystal and they vary notably within each unit cell.

High efficiency solar cells tend to be based on semiconductors with the zincblende structure, such as GaAs,InP, InAs, and alloys, among many others. For this structure, the basis usually considered comprises the involutes of the conduction band $(c b)$ and three valence bands: the light holes $(l h)$, the heavy holes $(h h)$ and the spin-orbit (so) band; all are spindegenerate. These are named $|\mathrm{S}\rangle$ for the $c b$, and $|\mathrm{X}\rangle,|\mathrm{Y}\rangle$ and $|\mathrm{Z}\rangle$ for the three valence bands. The latter three are degenerate at $\boldsymbol{k}=0$, and therefore cannot be associated specifically to $l h, h h$ or so. 
The basis using these involutes is called the standard basis in this paper. The Hamiltonian development in the mentioned basis leads to a four dimensional matrix Hamiltonian whose terms are functions of $\boldsymbol{k}$. In zincblende materials, this matrix is quite easy if the spinorbit interaction is neglected. In this easy form [10] we call it the zero-order Hamiltonian matrix $\left(H_{0}\right)$.

As explained in detail in references[11,12], we have defined the EKPH by its eigenvalues, which are the parabolic dispersion $E(\boldsymbol{k})$ functions for the $c b$, $h h$, $l h$ and so bands, defined by the experimental band edges and the effective masses. The use of these experimental dispersion functions takes into account the spinorbit interaction and any other forgotten effect that should have been taken into consideration. This is why we call it the empiric $\mathrm{k} \cdot \mathrm{p}$ Hamiltonian (EKPH). This Hamiltonian is known in its diagonalized form in a basis that we don't know how to relate to the standard basis. However, if we adopt the frequently used approximation of perturbing the energies and leaving the eigenvectors unperturbed, we assume that the new Hamiltonian $\left(H_{E K P}\right)$ has the same eigenvectors than $\left(H_{0}\right)$. In this case the diagonalizing matrix of $\left(H_{0}\right)$, called $(T)$, which is formed with the eigenvectors of $\left(H_{0}\right)$ is also valid to transform the standard basis into the unknown diagonalizing basis of $\left(H_{E K P}\right)$.

So far, the EKPH has been mainly applied to nanostructured solar cells. In QD solar cells, it has given reasonable agreement between calculated and measured sub-bandgap spectrum [18], and sub-bandgap quantum efficiency [11,12], including a good explanation of its temperature behavior [16]. In quantum well solar cells, it has given good agreement between the calculated and measured quantum efficiency [14]. With this work we want to further confirm the semi-quantitative adequacy of the method by checking the agreement for homogeneous materials.

The calculation of the envelope functions follows the following path [11,12]: (1) resolution of the diagonalized Time Independent Schrödinger Equation (TISE); (2) calculation of the solution's Fourier transform (plane wave development); (3) multiplication by the appropriate element of the transformation matrix (T); (4) calculation of the inverse Fourier transform.

\section{The Envelope Functions}

For a nanostructured semiconductor the diagonalized TISE is

$$
-\frac{\hbar^{2}}{2 m^{*}} \nabla^{2} \Phi(\boldsymbol{r})+V_{v}(\boldsymbol{r}) \Phi(\boldsymbol{r})=E \Phi(\boldsymbol{r})
$$

The asterisk denotes that we are considering the effective mass of the $c b$ or of the $l h, h h$, or so VBs. The offset energy of the band $v$ would act as potential energy $V_{v}(\boldsymbol{r})$ for nanostructured semiconductors. Bound functions are usually found for a discrete set of energies. The calculation of the envelope functions is obtained through the paths described above.

For homogeneous semiconductors, as is the case here, potential energy $V_{v}(\boldsymbol{r})$ is absent. Therefore the solutions are

$$
\Phi(\boldsymbol{r})=\exp \left(i \boldsymbol{k}^{\prime} \cdot \boldsymbol{r}\right) / \sqrt{\Omega}
$$

where the wavevector is the one chosen and the prime is to distinguish it from the $\boldsymbol{k}$ variable in the Fourier Transform used later. $\Omega$ is the volume of the space where the semiconductor is confined and is put in the wavefunction for normalization, The eigenenergy of an eigenfunction is (with respect to the band edge)

$$
E=\frac{\hbar^{2} k^{\prime 2}}{2 m^{*}}
$$

For VBs, the effective masses are negative and the equation must be written with the sign changed. This implies that the increasing energies start at the VB top and their values increase downwards.

The plane-wave development coefficients of $\Phi(\boldsymbol{r})$ in Eq. (3) can be directly written in terms of the Fourier Transform. In this case, all the functions are extended plane waves; we recall that $\mathcal{F}\left|e^{i k_{x}^{\prime} x}\right|\left(k_{x}\right)=\sqrt{2 \pi} \delta\left(k_{x}^{\prime}-k_{x}\right)$, therefore,

$$
\phi(\boldsymbol{k})=\left(\frac{2 \pi}{\Omega}\right)^{3 / 2} \delta\left(\boldsymbol{k}^{\prime}-\boldsymbol{k}\right)
$$

The elements of the transformation matrix (T) are presented in Figure 1.

In this figure $E_{g}$ is the semiconductor's bandgap, $m_{0}$ the mass of the electron in the vacuum and $m^{*}{ }_{c b}$ its effective mass in the conduction band. The parameter $\gamma$ is arbitrary. It reflects the fact that the $\left(H_{0}\right)$ matrix is double degenerate (additionally to the spin degeneration affecting to all bands) for the $h h$ and so bands; this constitutes a fundamental difference with the experimental dispersion curves for these bands, which are never degenerate (except for the spin degeneracy). Thus, for the $\left(H_{0}\right)$ matrix, there is a variety of possible $\left({ }^{\gamma} T\right)$ defined by $\gamma$. The summand matrices in the figure are separated for presentation purposes only. They have no physical meaning; separated, they are not unitary matrices, which is a necessary condition of the transformation matrices. $\left({ }^{\gamma} T\right)$ is a function of $\boldsymbol{k}$ through $\boldsymbol{K}=\boldsymbol{k} d$ where $d$ is an arbitrary normalizing length, (often $1 \mathrm{~nm}$ ). 


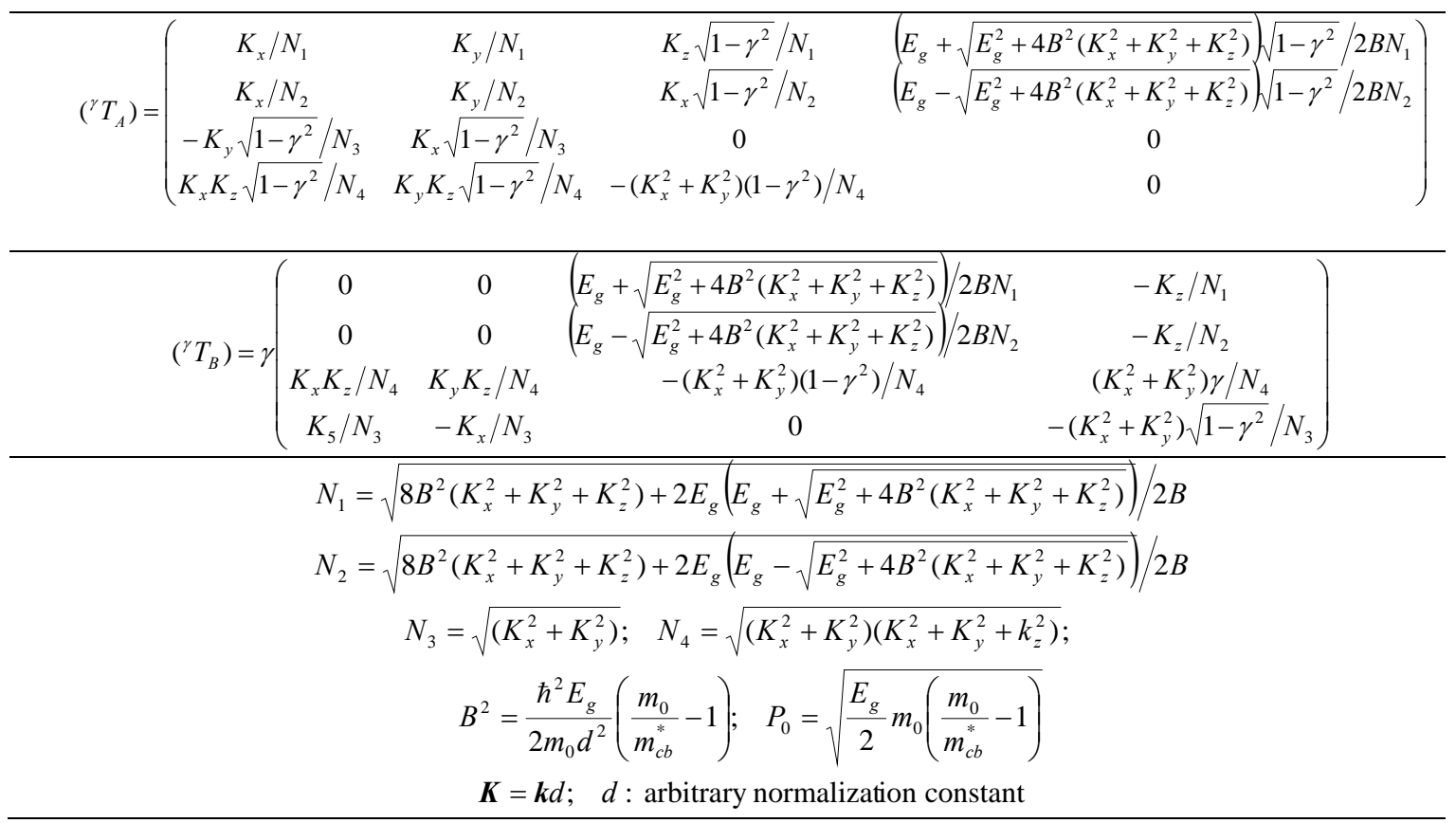

Figure 1. Summand matrices $\left({ }^{\gamma} T_{A}\right)$ and $\left({ }^{\gamma} T_{B}\right)$ into which the transformation matrix $\left({ }^{\gamma} T\right)$ is decomposed[12].

To calculate the envelope functions, $\phi(\boldsymbol{k})$ is to be multiplied by one of the elements ${ }^{\gamma} T_{i, j}(\boldsymbol{k})$. The row of the element, from 1-4, corresponds to the $c b, l h, h h$ and $s o$ bands respectively. The column of the element, form 1-4 corresponds to the $X$-, $Y$-, $Z$ - and $S$-envelopes respectively[12]. For example, the $Z$-envelope function of a light hole eigenfunction is obtained by multiplying the corresponding $\phi(\boldsymbol{k})$ by ${ }^{\gamma} T_{2,3}(\boldsymbol{k})$, also denoted ${ }^{\gamma} T_{\mathrm{lh}, \mathrm{Z}}(\boldsymbol{k})$.

Then, the envelope function is obtained by calculating the Inverse Fourier Transform of the function of $\boldsymbol{k}$ resulting from this product. Thus,

$$
\begin{aligned}
& \Psi(\boldsymbol{r})=\frac{(2 \pi)^{3 / 2}}{\Omega^{1 / 2}} \mathcal{F}^{-1}\left[{ }^{\gamma} T_{i, j}(\boldsymbol{k}) \delta\left(\boldsymbol{k}^{\prime}-\boldsymbol{k}\right)\right](\boldsymbol{r}) \\
& { }^{\gamma} T_{i, j}\left(\boldsymbol{k}^{\prime}\right) \frac{e^{i\left(\boldsymbol{k}^{\prime} \cdot \boldsymbol{r}\right)}}{\Omega^{1 / 2}}
\end{aligned}
$$

Note that all the envelopes are plane waves with the same wavevector than that of the diagonalized EKPH. The matrix elements ${ }^{\gamma} T_{i, j}(\boldsymbol{k}$ ') can be considered as the factor of projection of any plane wave on the corresponding involute. The sum of the square of their absolutes values is unity for any $\boldsymbol{k}$ '.

\section{The Dipole Matrix Elements}

Most nanostructures involve bound states. For transitions between electron states, one of which bound, with absorption or emission of one photon, we use $\langle\exists|\boldsymbol{\varepsilon} \boldsymbol{r}| \boldsymbol{\Xi}\rangle$, where $\boldsymbol{\varepsilon}$ is the light-polarization vector, as the dipole matrix element. The introduction of the radiation in the Hamiltonian leads to $\langle\Xi|\boldsymbol{\varepsilon} \boldsymbol{p}| \Xi\rangle$, where $\boldsymbol{p}$ is the momentum operator (proportional to the gradient), but $\langle\Xi|\varepsilon \cdot r| \Xi\rangle$ is obtained by application of the theorem of Ehrenfest.
However the operator $\boldsymbol{r}$ is not Hermitical at the infinity and $\langle\Xi \boldsymbol{\varepsilon} \boldsymbol{p} \mid \Xi\rangle[19,20,14]$ must be used for purely extended wavefunctions, as is the case here.

The application of $\boldsymbol{p}$ to a product of functions is $\boldsymbol{p}(u \Psi)=u \boldsymbol{p} \Psi+\Psi \boldsymbol{p} u$. Let us consider $\Psi$ to be slowly varying plane waves; this is true for small wavevectors. Due to the different scales associated to the $\mathrm{k} \cdot \mathrm{p}$ method, the integral factorization rule is considered applicable and $\left\langle u \Psi|\boldsymbol{\varepsilon} \boldsymbol{p}| u^{\prime} \Psi^{\prime}\right\rangle \cong\left\langle u \mid u^{\prime}\right\rangle\langle\Psi|\boldsymbol{\varepsilon} \boldsymbol{p}| \Psi\rangle+\left\langle u|\boldsymbol{\varepsilon} \boldsymbol{p}| u^{\prime}\right\rangle\langle\Psi$ $\left|\Psi^{\prime}\right\rangle[21,22,10]$. Thus

$\left\langle|\boldsymbol{\varepsilon} \cdot \boldsymbol{p}| \Xi^{\prime}\right\rangle=$

$\sum_{v}\left\langle\Psi_{v}|\boldsymbol{\varepsilon} \cdot \boldsymbol{p}| \Psi_{v^{\prime}}^{\prime}\right\rangle+\sum_{v, v^{\prime}}\left\langle u_{0, v}|\boldsymbol{\varepsilon} \cdot \boldsymbol{p}| u_{0, v^{\prime}}\right\rangle \Psi_{u}\left|\Psi_{u^{\prime}}^{\prime}\right\rangle$

where we have also considered that $\left\langle u_{0 v} \mid u_{0 v},\right\rangle=\delta_{v, v}$.

Any polarization orientation may be developed into its coordinate axes [12,23] leading to terms containing $p_{x}, p_{y}$, and $p_{z}$. For the calculation of $\left\langle u_{0 v}|\boldsymbol{\varepsilon} \boldsymbol{p}| u_{0 v}{ }^{\prime}\right\rangle$, we can use the symmetry properties of the $u$ s in zincblende crystals. The only non-null terms $[10,11]$ are

$$
\left\langle S\left|p_{x}\right| X\right\rangle=\left\langle S\left|p_{y}\right| Y\right\rangle=\left\langle S\left|p_{z}\right| Z\right\rangle=P_{0}
$$

where the link of $P_{0}$ with the experimental $m_{c b}^{*}$ may be found in Figure 1. Thus, for the case of $x$-polarization, Eq. (7) becomes

$$
\begin{aligned}
& \left\langle\Xi\left|p_{x}\right| \Xi^{\prime}\right\rangle=\Pi_{x}+P_{0}\left\langle\Psi_{S} \mid \Psi_{X}^{\prime}\right\rangle \\
& \Pi_{x} \equiv\left\langle\Psi_{X}\left|p_{x}\right| \Psi_{X}^{\prime}\right\rangle+\left\langle\Psi_{Y}\left|p_{x}\right| \Psi_{Y}^{\prime}\right\rangle \\
& +\left\langle\Psi_{Z}\left|p_{x}\right| \Psi_{Z}^{\prime}\right\rangle+\left\langle\Psi_{S}\left|p_{x}\right| \Psi_{S}^{\prime}\right\rangle \\
& \equiv \Pi_{x X}+\Pi_{x Y}+\Pi_{x Z}+\Pi_{x S}
\end{aligned}
$$

Vertical illumination involves photons with horizontal polarization. Let us examine, for example, $\Pi_{x S}$ for a $h h \rightarrow c b$ transition. It is a 
function of the differences $k_{x, h h}-k_{x, c v}=\omega_{x}$, $k_{y, h h} k_{y, c v}=\omega_{y}$ and $k_{z, h h}-k_{z, c v}=\omega_{z}$. Thus,

$$
\begin{aligned}
& \Pi_{x S}={ }^{\gamma} T_{c b, S}^{*}\left(\boldsymbol{k}_{c v}\right)^{\gamma} T_{h h, S}\left(\boldsymbol{k}_{h h}\right) \int_{-L_{z} / 2}^{L_{z} / 2} \frac{e^{i \omega_{z} z}}{L_{z}} d y \\
& \times \int_{-L_{y} / 2}^{L_{y} / 2} \frac{e^{i \omega_{y} y}}{L_{y}} d y \int_{-L_{x} / 2}^{L_{x} / 2} \frac{e^{i k_{c b, x} x} p_{x} e^{i k_{h h, x} x}}{L_{x}} d x
\end{aligned}
$$

where $\left(L_{x}, L_{y}, L_{z}\right)$ are the sides of the parallelepiped limiting the full volume $\Omega=$ $L_{x} L_{y} L_{z}$ which encloses the entire semiconductor. Under the usual periodic boundary conditions, the only permitted $k$ components are integer multiples of $2 \pi / L$ and the same for their differences $\omega$. The $y$ - and $z$-functions are one when $\omega_{y} \rightarrow 0$ and strictly zero for the permitted lateral differences, of $\pm 2 \pi / L, \pm 4 \pi / L$, etc. Thus, $k_{y}$ and $k_{z}$ are conserved. The $x$-function is $\hbar k_{h h, x}$ for $\omega_{x} \rightarrow 0$ and zero for the permitted lateral values. Thus, $k_{x}$ is also conserved. In summary, by dropping the band index (now useless) from the $k$-components,

$$
\Pi_{x S}=\hbar k_{x}{ }^{\gamma} T_{c b, S}^{*}(\boldsymbol{k})^{\gamma} T_{h h, S}(\boldsymbol{k})
$$

The same is applicable to other $\Pi$ terms. Therefore,

$$
\Pi_{x}=\hbar k_{x}\left(\begin{array}{c}
{ }^{\gamma} T_{c b, X}^{*}(\boldsymbol{k})^{\gamma} T_{h h, X}(\boldsymbol{k}) \\
+{ }^{\gamma} T_{c b, Y}^{*}(\boldsymbol{k})^{\gamma} T_{h h, Y}(\boldsymbol{k}) \\
+{ }^{\gamma} T_{c b, Z}^{*}(\boldsymbol{k})^{\gamma} T_{h h, Z}(\boldsymbol{k}) \\
+{ }^{\gamma} T_{c b, S}^{*}(\boldsymbol{k})^{\gamma} T_{h h, S}(\boldsymbol{k})
\end{array}\right)=0
$$

It is zero because the $(T)$ matrix is hermitic. Therefore, Eq. (7) can be rewritten as

$$
\left\langle\boldsymbol{k}^{\prime} \Xi_{c b}\left|p_{x}\right| \boldsymbol{k}^{\prime} \Xi_{h h}\right\rangle=P_{0}^{\gamma} T_{c b, S}^{*}\left(\boldsymbol{k}^{\prime}\right)^{\gamma} T_{h h, X}\left(\boldsymbol{k}^{\prime}\right)
$$

where we have put as a left-superindex the initially selected wavevector.

A similar treatment is to be used for $y$ - and $z$ - polarization. The $z$-polarization is not possible if the illumination is purely vertical.

\section{The absorption coefficients}

The number of photon absorptions per unit of time $[19,11]$ when the electron passes from state $|\Xi\rangle$ to state $\left|\Xi^{\prime}\right\rangle$ is

$$
\begin{aligned}
& w_{\Xi \rightarrow \Xi^{\prime}}=\Delta n_{p h} \frac{\pi e^{2} \hbar}{n_{r e f}^{2} m^{2} \varepsilon_{0} E} \\
& \times\left.|\Xi| \boldsymbol{p} \cdot \boldsymbol{\varepsilon}\left|\Xi^{\prime}\right\rangle\right|^{2} \delta\left(E_{\Xi^{\prime}}-E_{\Xi}-E\right)
\end{aligned}
$$

where $\varepsilon_{0}$ is the vacuum permittivity, $n_{\text {ref }}$ is the refractive index of the medium and $\Delta n_{p h}$ is the density of photons in all the modes of energy $E$. This density can be related to the photon flux by $\Delta F_{p h}=\left(c / n_{r e f}\right) \Delta n_{p h}$. Taking into account that the number of photon absorptions per unit of time is related to the elementary absorption coefficient $\alpha_{\Xi \rightarrow \Xi}$ in the transition under consideration by
$w_{\Xi \rightarrow \Xi^{\prime}}=\Delta F_{p h} \alpha_{\Xi \rightarrow \Xi^{\prime}}$, its expression is (for a $h h \rightarrow c b$ transition)

$$
\begin{aligned}
& \alpha_{\Xi \rightarrow \Xi^{\prime}}=\alpha_{\Xi \rightarrow \Xi^{\prime}}^{\prime} \delta\left(E_{\Xi}-E_{\Xi^{\prime}}-E\right) \\
& \text { with } \alpha_{\Xi \rightarrow \Xi^{\prime}}^{\prime}= \\
& \left.\left.\frac{\pi e^{2} \hbar P_{0}^{2}}{n_{r e f} m^{2} c \varepsilon_{0} E}\right|^{\gamma} T_{c b, S}^{*}(\boldsymbol{k})^{\gamma} T_{h h, X}(\boldsymbol{k})\right|^{2}
\end{aligned}
$$

Since all the transitions conserve $\boldsymbol{k}$, the transition energy $E_{t r}=E_{\Xi},-E_{\Xi}$ is given by

$$
\begin{aligned}
& E_{t r}=E_{g}+\frac{\hbar^{2} k^{2}}{2 m_{c o m b}^{*}}=E_{g}+E_{k i n} \\
& E_{g} \equiv E_{c b}-E_{h h} ; \quad \frac{1}{m_{c o m b}^{*}} \equiv\left(\frac{1}{m_{c b}^{*}}+\frac{1}{m_{h h}^{*}}\right)
\end{aligned}
$$

Note that, for holes, the energies in Eq. (4) must be reversed in sign and counted form the VB edge.

To calculate the total absorption coefficient, the elementary absorption coefficients per unit of volume must be added, that is $\alpha=(2 \pi)^{-3} \iiint_{\Xi \rightarrow \Xi^{\prime}} d k_{x} d k_{y} d k_{z} \cdot(2 \pi)^{-3}$ is the density of states per unit of 6D-volume in the $\left(x, y, \mathrm{z}, k_{x}, k_{y}, k_{z}\right)$ space. Thus,

$\alpha=\int_{E g}^{\infty} \frac{d}{d E}\left(\iiint_{D} \frac{\alpha^{\prime} d k_{x} d k_{y} d k_{y}}{(2 \pi)^{3}}\right) \delta\left(E_{t r}-E\right) d E$

with $D: k_{x}^{2}+k_{y}^{2}+k_{y}^{2} \leq 2 m_{\text {comb }}^{*} E_{\text {kin }} / \hbar^{2}$

where, using the expression of $P_{0}$ in Figure 1, and Eqs. (15) and (17),

$$
\begin{aligned}
& \iiint_{D} \frac{\alpha^{\prime} d k_{x} d k_{y} d k_{y}}{(2 \pi)^{2}}=2 \frac{e^{2} \hbar E_{g}\left(\left(m / m_{c b}^{*}\right)-1\right)}{16 \pi^{2} n_{r e f} m c \varepsilon_{0}} \\
& \times \iiint_{D\left(E_{k i n}\right)} \frac{\left|T_{c b, S}^{*}(\boldsymbol{k}) T_{h h, X}(\boldsymbol{k})\right|^{2}}{E_{k i n}+E_{g}} d k_{x} d k_{y} d k_{y}
\end{aligned}
$$

The first factor 2 corresponds to the spin degeneration.

In our calculations, the practical execution of the integral of Eq. (18) is done as follows:

The function inside the integral depends on the array of wavevectors $\left(k_{x}, k_{y}, k_{z}\right)$. We build an array containing in each term $\left\{E_{k i n}, \frac{\left|T_{c b, S}^{*}(\boldsymbol{k}) T_{h h, X}(\boldsymbol{k})\right|^{2}}{E_{k i n}+E_{g}}\right\}$, both elements being functions of the wavevector. The integral, restricted to a domain $D$, must be a function of the energy so that we must sum only those terms of $\frac{\left|T_{c b, S}^{*}(\boldsymbol{k}) T_{h h, X}(\boldsymbol{k})\right|^{2}}{E_{k i n}+E_{g}}$ such that their corresponding $E_{k i n}$ is smaller than a given value of $E_{k i n}$, building in this way a function of $E_{k i n}$. This function, which is rather smooth, is interpolated by a polynomial and its derivative is then taken for use in Eq. (17). 
We present in Figure 2 the results of these calculations together with a classic measurement [24]; similar measurement results may be found in other classic works $[17,25]$. The calculations have been made with the data in Table 1.

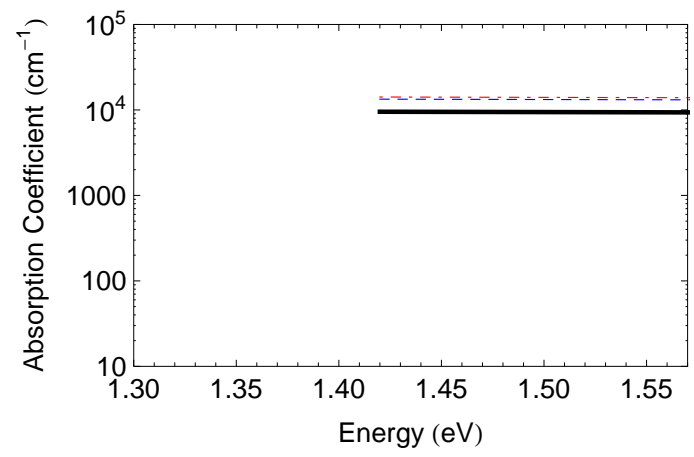

Table 1. GaAs parameters for calculations

\begin{tabular}{ll}
\hline \hline Bandgap GaAs (300 K) & 1.42 \\
$m_{c b} / m_{0}$ & 0.0613 \\
$m_{h h} / m_{0}$ & 0.35 \\
$m_{l h} / m_{0}$ & 0.09 \\
\hline \hline
\end{tabular}

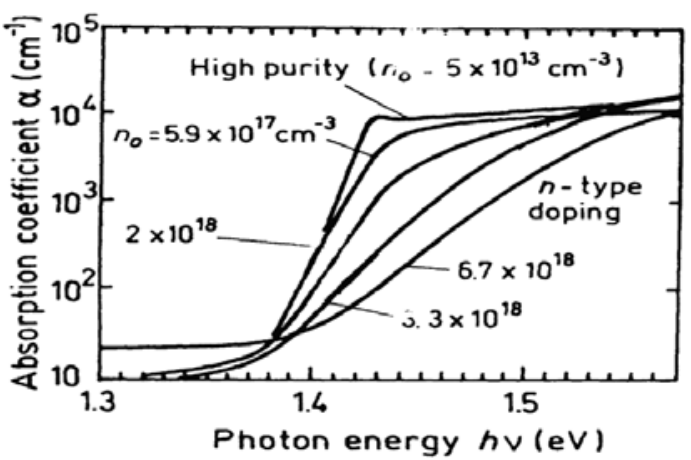

Figure 2. Left, calculated absorption coefficient for band to band transitions in GaAs for $\gamma=0$ (red, dotdashed line), $\gamma=2$ (blue, dashed line) and $\gamma=5$ (black, solid line). Right, measured absorption coefficient from[24], (c) 1975 AIP, with permission.

The curve to be compared is the one labeled "High purity". We can observe a reasonable agreement, although we must stress the semi quantitative nature we assign to them. The calculated result has been obtained in some few minutes with a laptop. This supports the adequacy of the use of the EKPH model.

The GaAs absorption coefficient takes values much higher, approaching or exceeding $10^{6} \mathrm{~cm}^{-1}$, for higher energies (e.g. $4 \mathrm{eV}$ ). This is due to transitions between more pairs of bands, which are enabled by the high photon energy. That subject is beyond of the scope of this work. Our calculations are limited to the transitions between the VB and CB.

\section{Conclusions}

In summary, the EKPH method has been applied to the calculation of the eigenfunctions and the band-to-band absorption of GaAs. The wavefunctions are linear combinations of plane waves multiplied by the $\Gamma$ point Bloch function (the involutes) for the four bands involved: $c b$, $l h, h h$ and $s o$. In this respect, they are close to the classical Bloch functions, in which the periodic part is dependent on the wavevector (and difficult to know), but they are not mixed with the other bands. The absolute value of the envelopes is given by the diagonalization element of matrix corresponding to the band of the state and to the concerned involute.

The optical absorption coefficients and the $\left\langle\Xi \boldsymbol{\varepsilon} \boldsymbol{p} \mid \Xi^{\prime}\right\rangle$ matrix elements can be calculated using only the envelopes, without making use of the involutes (thanks to the symmetry properties of the latter at the $\Gamma$ point); they depend only on the elements of the diagonalization matrix and their calculation is very simple. The calculations are very fast and the results are reasonably accurate.

In all, the results give an additional proof of the adequacy of the EKPH model.

\section{Acknowledgment}

This work has been sponsored by the Megagrant No. 14B25.31.0020 from the Russian Ministry of Education and Science and by the European Commission through the NGCPV Project (Grant No. 283798).

\section{References}

1. Dresselhaus, G., Kip, A.F., Kittel, C.: Plasma resonance in crystals - observations and theory. Physical Review 100(2), 618-625 (1955). doi:10.1103/PhysRev.100.618

2. Kane, E.O.: Energy band structure in p-type germanium and silicon. Journal of Physics and Chemistry of Solids 1(12), 82-99 (1956). doi:10.1016/00223697(56)90014-2

3. Kane, E.O.: The $\mathrm{k} \cdot \mathrm{p}$ method. In: Willardson, R.K., Beer, A.C. (eds.) Physics of III-V Compounds. Semiconductors and Semimetals, vol. 1, pp. 75-100. Academic, New York (1966)

4. Bastard, G.: Wave Mechanics Applied to Semiconductor Nanostructures. Monographies de Physique. Les Editions de Physique, Paris (1990)

5. Basu, P.K.: Theory of Optical Processes in Semiconductors. Series on Semicoductor Science and Technology. 
Oxford University Press, Oxford, UK (1997)

6. Wang, L.W., Zunger, A.: Electronic-structure pseudopotential calculations of large (approximate-to-1000 atoms) Si quantum dots. Journal of Physical Chemistry 98(8), 2158-2165 (1994). doi:10.1021/j100059a032

7. Ivchenko, E.L.: Optical Spectroscopy of Semiconductors Nanostructures Alpha Science, Harrow (UK) (2005)

8. Voon, L.C.L.Y., Willatzen, M.: The $\mathrm{k} \cdot \mathrm{p}$ method. Electronic Properties of Semiconductors. Springer, Berlin (2009)

9. Galeriu, C.: K·P theory of semiconductor nasnostructures. $\mathrm{PhD}$ Disertations. Worcester Polytechnic Institute, Worcester (2005)

10. Datta, S.: Quantum Phenomena. Molecular Series on Solid State Devices, vol. 8. Addison Wesley, Reading (Mass) (1989)

11. Luque, A., Marti, A., Antolín, E., Linares, P.G., Tobías, I., Ramiro, I., Hernandez, E.: New Hamiltonian for a better understanding of the Quantum Dot Intermediate Band Solar Cells. Solar Energy Materials \& Solar Cells 95, 2095-2101 (2011). doi:doi:10.1016/j.solmat.2011.02.028

12. Luque, A., Mellor, A., Antolin, E., Linares, P.G., Ramiro, I., Tobias, I., Marti, A.: Symmetry considerations in the empirical k.p Hamiltonian for the study of intermediate band solar cells. Solar Energy Materials and Solar Cells 103(0), 171-183 (2012).

13. Luque, A., Mellor, A., Ramiro, I., Antolín, E., Tobías, I., Martí, A.: Interband absorption of photons by extended states in intermediate band solar cells. Solar Energy Materials \& Solar Cells 115, 138-144 (2013).

14. Luque, A., Antolín, E., Linares, P.G., Ramiro, I., Mellor, A., Tobías, I., Martí, A.: Interband optical absorption in quantum well solar cells. Solar Energy Materials \& Solar Cells 112, 20-26 (2013). doi:DOI: 10.1016/j.solmat.2012.12.045

15. Luque, A., Linares, P.G., Mellor, A., Andreev, V., Marti, A.: Some advantages of intermediate band solar cells based on type II quantum dots.
Applied Physics Letters 103, 123901 (2013). doi:10.1063/1.4821580

16. Mellor, A., Luque, A., Tobías, I., Martí, A.: Realistic detailed balance study of the quantum efficiency of quantum dot solar cells. Advanced Functional Materials 24(3), 339-345 (2014). doi:10.1002/adfm.201301513

17. Hobden, M.V., Sturge, M.D.: Optical Absorption Edge of Gallium Arsenide. Proceedings of the Physical Society of London 78(502), 615-\& (1961). doi:10.1088/0370-1328/78/4/119

18. Canovas, E., Fuertes Marron, D., Marti, A., Luque, A., Bett, A.W., Dimroth, F., Philipps, S.P.: Photoreflectance analysis of a GaInP/GaInAs/Ge multijunction solar cell. Applied Physics Letters 97(20), 203504 (2010). doi:10.1063/1.3517255

19. Messiah, A.: Mécanique Quantique. Dunod, Paris (1960)

20. Luque, A., Marti, A., Mendes, M.J., Tobias, I.: Light absorption in the near field around surface plasmon polaritons. Journal of Applied Physics 104(11), 113118 (2008). doi:10.1063/1.3014035

21. Harrison, P.: Quantum Wells Wires and Dots. John Wiley and Sons, New York (2000)

22. Coon, D.D., Karunasiri, R.P.G.: New mode of IR detection using quantum wells. Applied Physics Letters 45(6), 649-651 (1984). doi:10.1063/1.95343

23. Luque, A., Marti, A., Antolín, E., Linares, P.G., Tobias, I., Ramiro, I.: Radiative thermal escape in intermediate band solar cells. AIP Advances 1, 022125 (2011).

24. Casey, H.C., Sell, D.D., Wecht, K.W.: Concentration-Dependence of Absorption-Coefficient for n-Type and p-Type GaAs between 1.3 and $1.6 \mathrm{eV}$. Journal of Applied Physics 46(1), 250257 (1975). doi:10.1063/1.321330

25. Sturge, M.D.: Optical Absorption of Gallium Arsenide between 0.6 and 2.75 eV Physical Review 127(3), 768\& doi:10.1103/PhysRev.127.768
(1962). 